\title{
In vivo confocal Raman spectroscopy and molecular dynamics analysis of penetration of retinyl acetate into stratum corneum
}

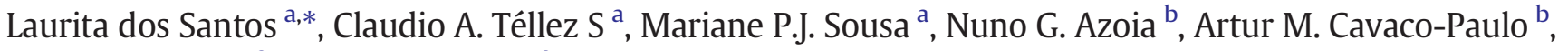 \\ Airton A. Martin ${ }^{a}$, Priscila P. Favero ${ }^{a}$ \\ a Laboratory of Biomedical Vibrational Spectroscopy, University of Vale do Paraíba, 12224-000 São José dos Campos, SP, Brazil \\ b Centre of Biological Engineering, University of Minho, 4710-057 Braga, Portugal
}

\section{A R T I C L E I N F O}

Article history:

Received 8 August 2016

Received in revised form 17 November 2016

Accepted 26 November 2016

Available online 05 December 2016

\section{Keywords:}

In vivo confocal Raman spectroscopy

Molecular dynamics

Stratum corneum

Retinyl acetate

Skin penetration

\begin{abstract}
A B S T R A C T
Objective: The purpose of this study is to elucidate the behavior of retinyl acetate in penetrating human skin without the presence of enhancers by using confocal Raman spectroscopy and molecular dynamics simulation.

Methods: In this study, in vivo confocal Raman spectroscopy was combined with molecular dynamics simulation to investigate the transdermal permeation of the aqueous suspension of retinyl acetate.

Results: Permeation was measured after $30 \mathrm{~min}$, and retinyl acetate was found up to $20 \mu \mathrm{m}$ deep inside the stratum corneum. The delivery of retinyl acetate inside a skin membrane model was studied by molecular dynamics. The membrane model that was used represented normal young skin containing a lipid bilayer with $25 \%$ ceramide, 36\% fatty acid, 30\% cholesterol, and 6\% cholesterol sulfate.

Conclusion: Spectroscopy data indicate that retinyl acetate permeates into the stratum corneum. Molecular dynamics data showed that retinyl acetate permeates in the membrane model and that their final location is deep inside the lipid bilayer. We showed, for the first time, a correlation between Raman permeation data and computational data.
\end{abstract}

C 2016 Elsevier B.V. All rights reserved.

\section{Introduction}

According to several authors, vitamin A and its derivatives provide topical protection against the effects of photoaging in skin $[1,2]$ and severe acne [3-6], and they may improve skin elasticity by increasing the number and activity of fibroblasts [6]. This vitamin is significantly absorbed by human skin when it is applied with water in an oil emulsion or in a hydro-alcoholic gel. Among the vitamin A derivatives, retinyl acetate (RA) is better tolerated by skin than retinol is [7]. RA is the vitamin A derivative that is most common in cosmetic products, and it can be encapsulated for skin application [8].

The first barrier for RA transdermal permeation is the stratum corneum (SC), which is the outer layer that provides the main obstacle against microorganisms and water loss [9]. The SC thickness varies according to body site. In the cheek region, the SC thickness is $12 \mu \mathrm{m}$ to $16 \mu \mathrm{m}$; in the back region, it is approximately $29 \mu \mathrm{m}$; and in the volar forearm region, it is $18 \mu \mathrm{m}$ to $22 \mu \mathrm{m}$ [10,11]. The intercellular lipid lamellae of the SC may be represented by a lipid bilayer that is predominantly composed of ceramides, cholesterol, and fatty acids [12-14].

\footnotetext{
* Corresponding author.

E-mail address: lauritas9@gmail.com (L. dos Santos).
}

The composition of the lipid bilayer dictates the affinity and transport of the vitamin through membrane [15].

The penetration process and molecule transport are usually studied by complementary techniques, such as in vivo experiments and computational simulations [13-17]. Molecular dynamics simulations reproduce the behavior of membranes [18] in a large-scale temporal approach [19], particularly when using a coarse grained (CG) molecular approach [20-22]. Martins et al. recently elaborated a cosmetic nano formulation for in vitro transdermal permeation analysis. Using molecular dynamics, they confirmed the permeation of the formulation into the lipid bilayer in silico model [23]. Other approaches, such as in vivo confocal Raman spectroscopy, may be combined with in silico techniques to understand the permeation process of compounds in human skin.

In vivo confocal Raman spectroscopy is a powerful non-invasive technique that allows an analysis of skin constituents [24-29] related to biochemical composition, permeation processes [27-30] of the skin and RA, and it can replace other methods such as skin biopsy and tape tripping [31,32].

Confocal Raman equipment can detect skin profiles from the surface to a depth of $175 \mu \mathrm{m}$ [33]. This technique is used to investigate the permeation of enhancers on trans-retinol delivery into skin by using different formulations, as described previously [26,34]. The combination of in vivo and in silico techniques is an alternative method that corroborates 
the understanding of some delivery processes by developing a new technology or methodology, such as drug delivery [35].

In this study, in vivo confocal Raman spectroscopy was combined with molecular dynamics simulation to investigate the penetration of retinyl acetate to elucidate the behavior of RA permeating into human skin without the presence of enhancers.

\section{Methodology}

To elucidate the behavior of RA without any interference, water was used as a vehicle in a suspension with $0.015 \%$ retinyl acetate (500.000 IU/g feed grade; Xiamen Kingdomway Vitamin Corporation, Xiamen, China). An ultrasonic bath (40 kHz, model USC 1450, Unique, Brazil) was applied for $30 \mathrm{~min}$ to obtain homogeneity. The particle size was measured using a Particle Size Analyzer Microtrac model S3550. The particle size analyzer system can detect a minimum aggregate particle size of $0.0255 \mu \mathrm{m}$ but cannot detect individual molecules of retinyl acetate. These procedures (ultrasonic bath + particle size measurement) were always repeated before application on the forearm skin of the study participant.

\subsection{Study Participants}

Five healthy study participants (Caucasian women, $30 \pm 7$ years old) were selected, with Fitzpatrick phototype I or II [27], following the written agreement. The selection of skin phototype I and II was based on the scattering technique, where the presence of melanin is associated with the fluorescence effect. This effect has a significant impact on the quality of the spectra for in vivo analysis. The exclusion criteria for study participants were a history of dermatological disease, pregnancy or lactation, sensitivity to cosmetic components, and if they had used any type of cosmetic product on the forearm skin within $24 \mathrm{~h}$ before the experiment. The study participant washed (with neutral soap and water) the area (approximately $1 \mathrm{~cm}^{2}$ ) of forearm that was to be measured and waited for $20 \mathrm{~min}$ in an air-conditioned environment at $23 \pm 1{ }^{\circ} \mathrm{C}$ and a relative humidity of $50 \pm 5 \%$. The suspension (approximately $5 \mathrm{ml}$ ) was applied on the participant's forearm. Before moving the arm, the participant waited for the water in the suspension to evaporate/disappear. The principal role of the water was to facilitate the application of the RA onto the skin. The RA interacts with skin because of its liposolubility.

The study was approved by the Research Ethics Committee (CEP) under protocol number 09112612.7.0000.5503. All study participants signed a consent form before the start of the study and received information about the study procedures.

\subsection{Confocal Raman Spectroscopy}

The acquisition of Raman spectra were performed using a confocal Raman system 3510 Skin Composition Analyzer (River Diagnostics, Rotterdam, The Netherlands), with the excitation laser set at $785 \mathrm{~nm}$, the power at the sample set at of $26 \mathrm{~mW}$ and $2 \mu \mathrm{m}$ of spatial resolution in the $z$-direction. The calibration measurements of the Raman system were performed according to the short guide of Model 3510 SCA provided by River Diagnostics. This process involves many set-up steps, including the CCD camera, neon/argon calibration standard, Raman fingerprint region, background fingerprint in (and above) window and NIST standard calibrations. These standard calibrations were obtained by the default setup of the spectrometer. The Raman data were collected by a CCD detector (see Fig. 1 ).

The fingerprint (400-1800 $\mathrm{cm}^{-1}$ ) region was analyzed at different depths ( 0 to $20 \mu \mathrm{m}$ ), with a step size of $2 \mu \mathrm{m}$. The integration time was $10 \mathrm{~s}$ for each depth. The average Raman spectrum was calculated for each depth profile.

To evaluate the retinyl acetate permeation through the stratum corneum, the study was divided into two sets of measurements, T0

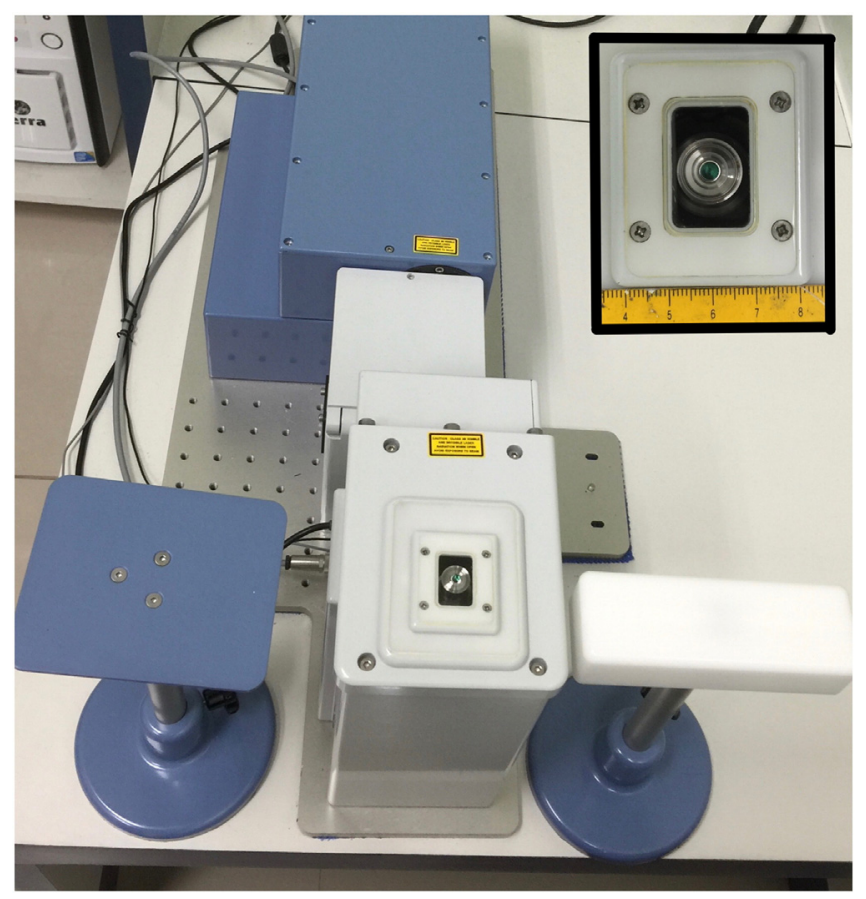

Fig. 1. Confocal Raman system model 3510 showing in detail the CCD detector.

and T30. T0 represents the confocal Raman spectra before the vitamin A derivative in water was applied on the skin. T30 represents the confocal Raman spectra measurements from the same region obtained 30 min after the RA application.

Before each measurement, the forearm was gently wiped with optic paper to remove any excess RA from the surface of the skin. Each measurement (T0 and T30) was taken at three different points of the forearm region. To analyze the permeation process, we computed the difference between $\mathrm{T} 0$ and $\mathrm{T} 30$ by using the averaged spectrum for each depth of the participant's epidermis.

To pre-process the Raman spectra data, the polynomial baseline correction method was used. The background was estimated by a 5 degree polynomial using the LabSpec $5.78 \circledR$ software (Horiba) and was normalized by the amide I band using Origin $8.5 \AA$.

\subsection{Molecular Dynamics}

\subsubsection{Stratum Corneum Model}

The elaborated stratum corneum model is based on the molecular constituents of normal young human skin [12] and on previously published simulation data [13], i.e., ceramide-2 (25\%), fatty acid (36\%), cholesterol (30\%) [9] and cholesterol sulfate (6\%) [14]. These lipids all play an important role in the model. Ceramide-2 (ceramide NS) retains substantial ordering [9] and is responsible for the low water permeability of the stratum corneum [36]. The fatty acid is significantly more mobile than the ceramide [9]. The role of the cholesterol allows fluidity for the lipid bilayer [37], and the cholesterol sulfate facilitates and stabilizes the formation of the lipid lamellae [38].

The model was built using the CELLmicrocosmos 2.2 package [39]. The molecules involved in this coarse-grained model were obtained according to Martins et al. 2013 [23]. The size of the box simulation obtained was $15 \times 15 \times 12 \mathrm{~nm}^{3}$. The negative charges were neutralized with $366 \mathrm{Na}^{+}$.

The simulations were executed using the GROMACS 4.5.5 package [40] with the Martini [20] forcefield, applying periodic boundary conditions in all dimensions, i.e., $x, y$, and $z$. The bond lengths were restrained using the LINCS algorithm [41]. The cut-offs for the Van der Waals and electrostatic interactions were adjusted to $1.2 \mathrm{~nm}$ [9]. A neighboring search was conducted up to $1.2 \mathrm{~nm}$ and was updated every ten steps. 


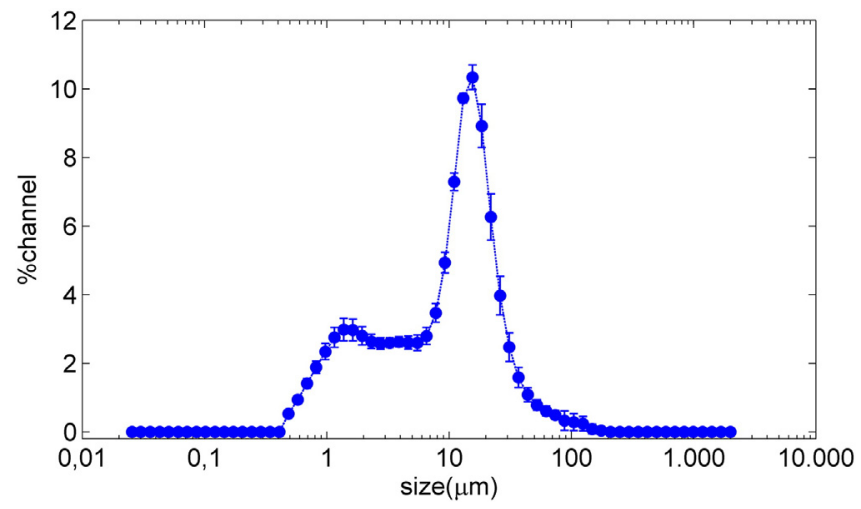

Fig. 2. Distribution (average \pm standard deviation) of the particle size for retinyl acetate in water after $30 \mathrm{~min}$ of the ultrasonic bath.

The integration time was $20 \mathrm{fs}$. A Berendsen barostat with a reference pressure of 1 bar [42] was used for pressure coupling, with a relaxation time of $3.0 \mathrm{ps}$ and an isothermal compressibility of $3.0 \times 10^{-5} / \mathrm{bar}$. A $v$ rescale thermostat [43] at $300 \mathrm{~K}$ [23] was used for temperature control. Each system component was included separately in contact with heat, with temperature coupling constants of $1.5 \mathrm{ps}$.

The stratum corneum model was considered to be converged after 500 ns of the simulation, and it was applied as the basic model for permeation studies.

\subsubsection{Retinyl Acetate Model}

First, it was necessary to parameterize the vitamin A derivative to develop a CG model (fine-grained to coarse-grained representation) [44]. The molecule was divided into small chemical groups or blocks (around four atoms, disregarding the hydrogen atoms), and each atomic group was mapped as one new CG particle. The RA $\left(\mathrm{C}_{22} \mathrm{H}_{32} \mathrm{O}_{2}\right)$ turned seven particles, where the functional acetate group was parameterized as a CG polar group and other CG particles were parameterized as an apolar group. The guide to CG particle type was presented by Marrink S.J et al. [20]. This parameterization maintained the main characteristics of the molecule.

The RA was optimized separately, following the same parameters used for the model of the lipid bilayer. The integration time interval was changed to 5 fs.

\section{Results and Discussion}

The main purpose of the water as a vehicle was to allow the suspension of the isolated retinyl acetate on the skin without any interference from excipients. The hydrophilic part of the RA is surrounded by water molecules. This effect causes the perpendicular orientation of the apolar part of the active principle on the skin surface, thus permitting its permeation.

The ultrasonication ( $30 \mathrm{~min}$ ) treatment of the suspension reduced the granularity of the vitamin A derivative for stratum corneum penetration. Particles with $1 \mu \mathrm{m}$ diameters have more contact with water molecules, which is conducive to disaggregation and permits their individual permeation. The mean values of the summary peak maxima provided by the particle size analyzer represent $98.7 \%$ of the suspension volume. The remaining $1.3 \%$ of the volume must contain aggregates with different sizes, where those of the lowest size have a greater possibility of interacting with the skin surface for permeation.

Fig. 2 shows the average and standard deviation of the particle size distribution. The peak summary provides predominantly two particle sizes: $12.0 \pm 0.7 \mu \mathrm{m}$, which corresponds to $87 \pm 9 \%$ of the total volume, and $1.09 \pm 0.06 \mu \mathrm{m}$, which corresponds to $18 \pm 3 \%$ of the volume. The difference in the particle sizes implies the heterogeneous characterization of the suspension.

\subsection{In Vivo Experimental Studies}

To analyze the permeation of the active principle through the skin epidermis by confocal Raman spectroscopy, the applied substance must have some non-coincident Raman bands with the Raman spectra of the skin epidermis. Fig. 3 displays the raw Raman spectra (T0 and T30 measurements) without any pre-processing data. For the RA, the most prominent non-coincident Raman band was centered at $1593 \mathrm{~cm}^{-1}$ (dashed region in Fig. 4). This shoulder was observed at different depths, which suggested that RA penetrated the skin.

In the Raman spectra of the skin, we found characteristic bands of the amino acid chain assigned to the lipoprotein constitution (1580 $\mathrm{cm}^{-1}$ to $1720 \mathrm{~cm}^{-1}$ region), where the peptide bonds -C[C=O]-NH-R exist.

The wavenumber position of the $\nu(\mathrm{C}=0)$ of the retinyl acetate with reference to the Amide I band wavenumber can be mostly explained by the resonance effect through the ether and carbonyl atom of the R-O$(\mathrm{C}=\mathrm{O})-\mathrm{OH}$ structural fragment. The $\mathrm{C}=\mathrm{O}$ bond and the force constant

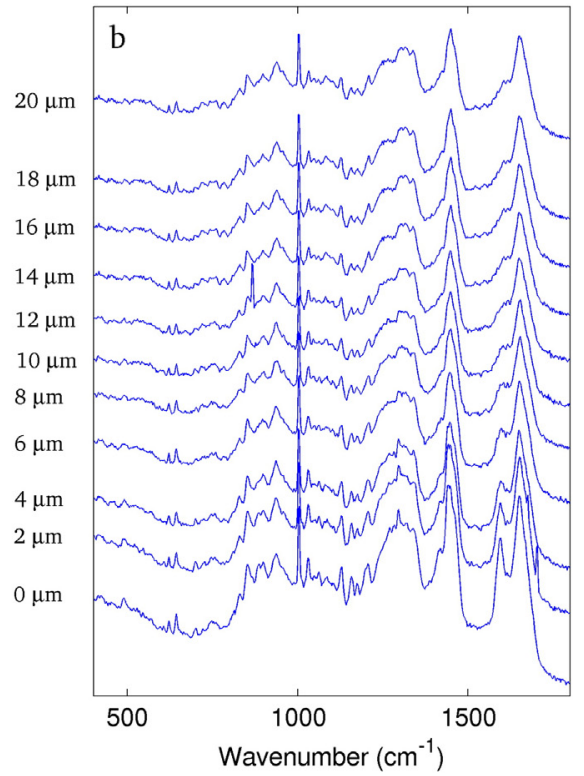

Fig. 3. Raw data a) T0 and b) T30 measurement without pre-processed analysis. 

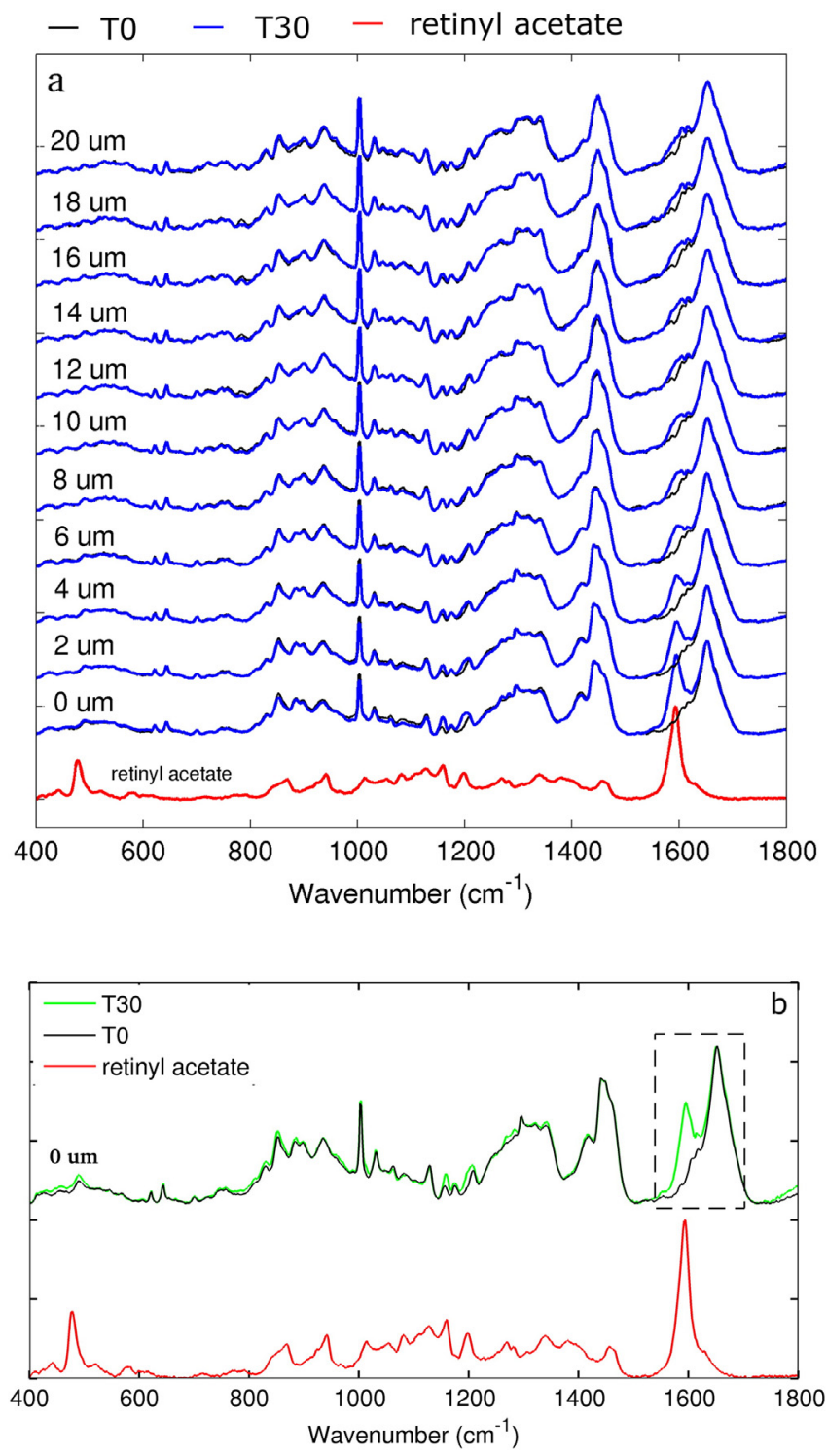

Fig. 4. Profile of the Raman spectra for skin surface up to depth of $20 \mu \mathrm{m}$ of the skin, a) T0 and T30 measurements for all depths, b) dotted line represents the pure Raman spectrum of the RA. Dashed region is the main difference between T0 and T30 spectra.

of the carbonyl bond become weaker upon acquiring the oxygen atom negative charge [46].

To determine the extended penetration of the RA into the skin, the intensity of the $1593 \mathrm{~cm}^{-1}$ Raman band was determined for different depth profiles in the skin. To perform such a calculation, we consider the band area at $1593 \mathrm{~cm}^{-1}$ in $\left[\mathrm{R}_{\text {int. }}\right.$ (arbitr. unit) $\times \mathrm{cm}^{-1}$ ] of the RA control sample to have a value of $100 \%$ (see Fig. 5 ). The peaks in the skin spectrum displayed at $1606 \mathrm{~cm}^{-1}$ and $1615 \mathrm{~cm}^{-1}$ are not changed in terms of intensity as a function of the increasing depth.

The other intensity values were obtained from the relative area of this band after 30 min (T30) of RA application with the control sample from the surface to $20 \mu \mathrm{m}$ deep in the skin.

Fig. 6 corresponds to the graphical representation of the exponential decay function $\mathrm{y}=A_{1} * \mathrm{e}^{(-\mathrm{x} / \mathrm{t} 1)}+\mathrm{y}_{0}$, where $\mathrm{R}^{2}=0.97533$ and $p<0.0001$. The parameter $A_{1}$ is related to the amplitude (quantity of product on the skin surface), and $t_{1}$ is the decay constant associated with absorption coefficient of the RA penetration into skin. Interpreting the angular coefficient of the curve for a skin profundity of 0 gives -0.28 [area (a.u.) $x \mu \mathrm{m}$ ]; thus, the instantaneous velocity of the variation of RA penetration at zero profundity is given by $-\mathrm{d}[S P] /$ $\mathrm{d}[$ area] $=0.28$ [area (a.u.) $x \mu \mathrm{m}]$. This result is evidence of the high affinity of retinyl acetate for lipids and of its mobility into the stratum corneum.

\subsection{Molecular Dynamics Data}

The stabilization of the stratum corneum model is shown in Fig. 7. The simulation of the lipid bilayer was extended at $500 \mathrm{~ns}$, and the variation of the potential energy $(\mathrm{kJ} / \mathrm{mol})$ is constant after $100 \mathrm{~ns}$. This time extension allows a suitable stratum corneum model that can interact with the chosen active principle.

The computational permeation model analyzes the interaction of retinyl acetate molecules and the lipid bilayer. The box simulation contained 11,352 molecules, 1433 lipids present in the stratum corneum, 9909 CG molecules of water (each water CG particle corresponds to four individual molecules of the water), and ten molecules of vitamin A derivatives. We considered that the disaggregation of the RA microparticles obtained with (see Subsection 3.1) led to an individual permeation of the active principle in the skin.

The time evolution of simulation is shown in Fig. 8. The first snapshot shows that ten RA molecules are scattered in the box simulation. After approximately 30 ns of the simulation, all of these molecules penetrated into the first lipid layer. The permeation process was a result of the high chemical affinity of the active principle to the lipids (see Fig. 5). The apolar region (large blue spheres in Fig. 8) of RA was the first part that penetrated into the lipid layer. The polar part (two last green spheres in tail of retinyl acetate) was the last to penetrate.

The simulation was extended to $160 \mathrm{~ns}$, and at this time point, the system reached a stable configuration. Significant differences were not observed between the snapshots taken at 30 and 150 ns. The cholester$\mathrm{ol}$, in which the head group is represented by a small sphere among lipid tails as shown in Fig. 8, flowed into the lipid bilayer, as displayed in Fig. 9. This behavior of cholesterol translocation was previously described in Choubey 2013 et al., who showed the flip-flop dynamics into a dipalmitoylphosphatidylcholine (DPPC) bilayer [45].

The RA mobility into the bilayer (red circle region in Fig. 8) was noted at approximately $157 \mathrm{~ns}$. The mobility was related to the RA solubility in the lipid bilayer and its carbon chain. Although there was a polar group (acetate group) in the active principle, the extension of the apolar region allowed RA translocation into the lipid matrix.

The density profile (Fig. 9) represents the relative position of the components with respect to the stratum corneum model at the beginning and end of the simulation. The system was pre-equilibrated prior to the interactions with RA; therefore, it is not expected that the lipid distribution at the bilayer will change significantly. This observation corroborates the fact that Raman spectra do not display changes in the lipid composition at different depths of the stratum corneum layer.

In fact, the ceramides, water, and cholesterol did not present large differences between the initial and final density profiles. The small changes presented are consistent with a swelling of the bilayer to accommodate the FA molecules. On the contrary, at the end of the simulation, the density profile of the retinyl acetate changed dramatically, showing the penetration of these molecules deep into the lipid bilayer. From the literature, it is considered that the penetration of active components to the lipid bilayer shows good experimental agreement according to molecular dynamics [23].

\section{Conclusions}

This study presented the skin permeation of retinyl acetate using experimental and computational techniques. In vivo confocal Raman spectroscopy is a powerful non-invasive technique that can analyze skin constituents and the permeation of formulations into the skin. Our results show that the intensity band of the RA (approximately $1593 \mathrm{~cm}^{-1}$ ), representing the $-\mathrm{C}=0$ vibrational mode, was detected 

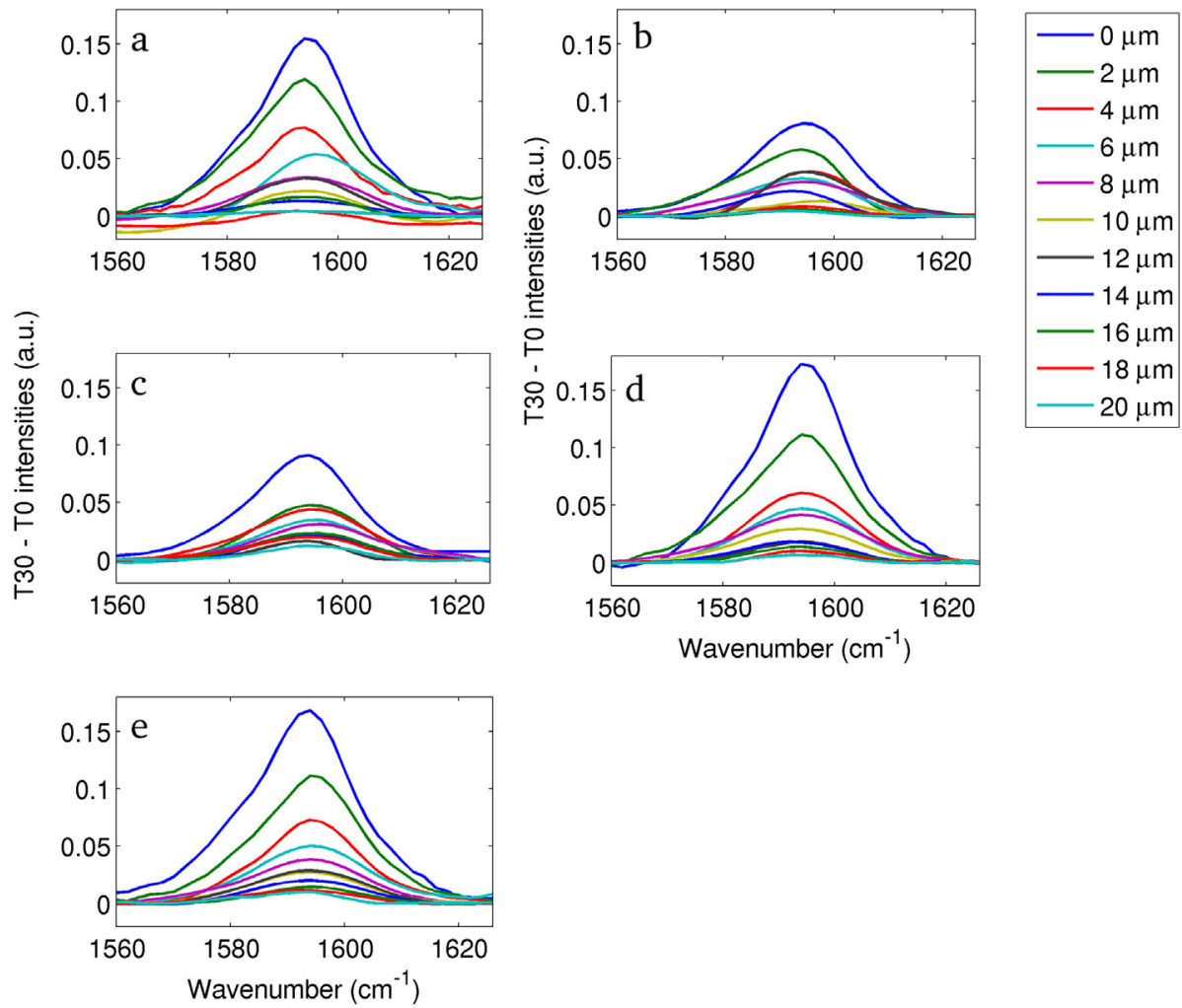

Fig. 5. Relative intensity of the RA band at $1593 \mathrm{~cm}^{-1}$ obtained by subtraction of the T30 and T0 measurements for five study participants.

in the Raman spectra of skin at different stratum corneum depths up to $20 \mu \mathrm{m}$. This Raman peak of retinyl acetate has a non-coincident band with the Raman spectra of the skin epidermis, demonstrating that the vitamin A derivative penetrated forearm skin.

We demonstrated retinyl acetate translocation using an in silico model. The translocation is directly associated with the ability of RA to migrate into the lipidic bilayer and is a good simulation of skin permeation. This was confirmed by the observation of the translocation phenomenon that is related to a different Raman intensity of the vibrational mode $\nu(\mathrm{C}=0)$, where its intensity was found in up to $20 \mu \mathrm{m}$ deep. Thus, experimental and computational techniques may be combined to understand the penetration process through the skin stratum corneum, and the computational results agree well with the experimental ones.

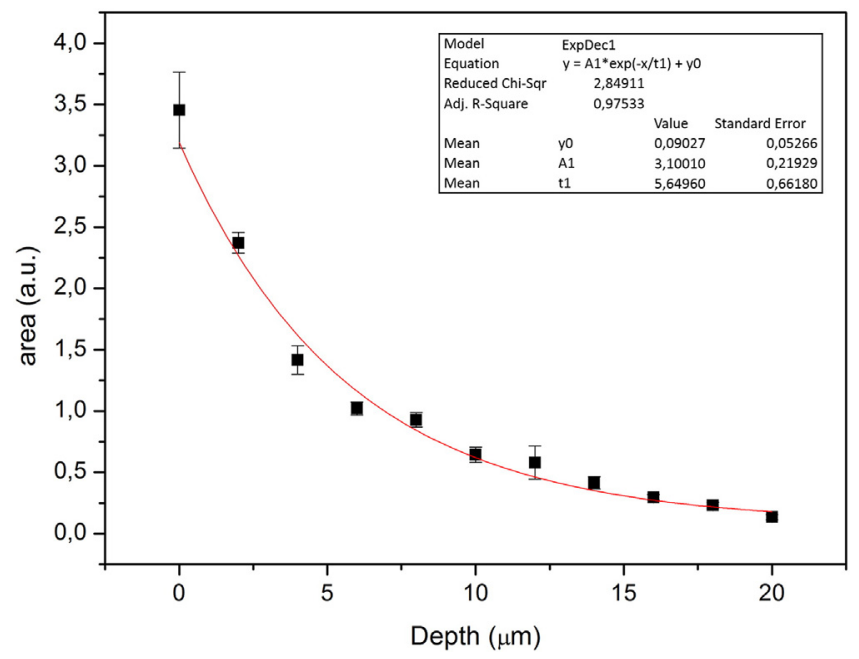

Fig. 6. Area values (a.u.) (average and standard deviation) for skin surface up to $20 \mu \mathrm{m}$ depth into the skin.

\section{Conflict of Interest}

The authors declare that there is no conflict of interest regarding the publication of this manuscript.

\section{Acknowledgement}

We thank CNPq for their financial support (A.A. Martin 307809/ 2013-7). The particle size measurement was performed by Laboratório Multi-Usuário, UniVap. We are grateful to IP\&D - UniVap cluster of computers (FINEP 01.10.0661-00, Fapesp 2011/13250-0, and Fapesp 2013/ 17247-9). L. dos Santos would like to thank Capes/Brazil (88887.068264/2014-00 and 88881.062862/2014-01) for their funding.

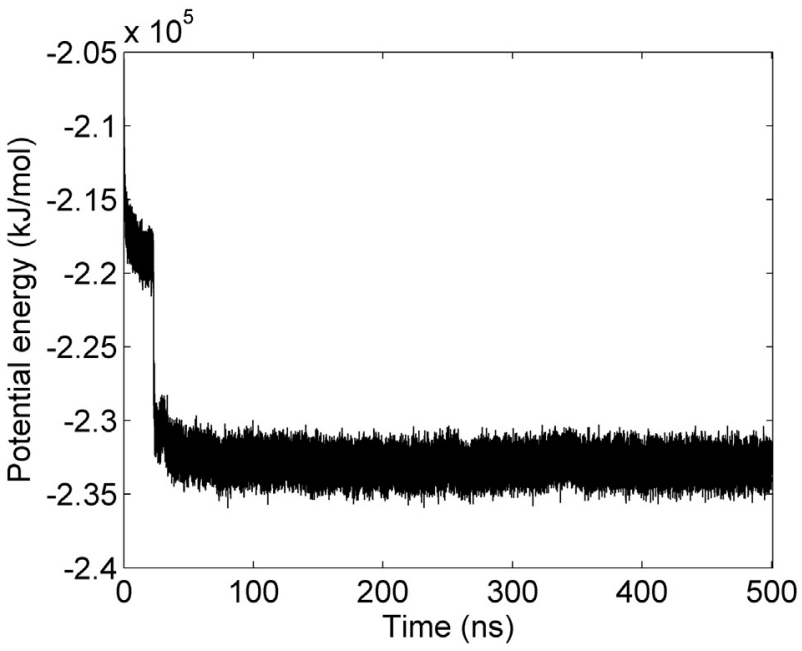

Fig. 7. Variation of potential energy $(\mathrm{kJ} / \mathrm{mol})$ of the lipid bilayer model at $500 \mathrm{~ns}$. After $100 \mathrm{~ns}$, the variation is constant. This indicates that the lipid bilayer (system) reached the energy equilibration. 


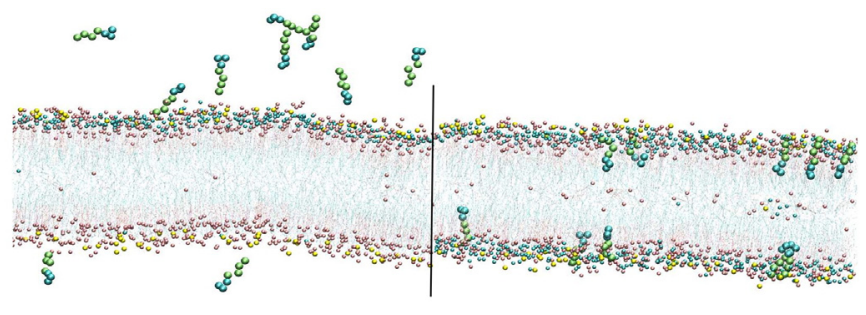

0 ns

$30 \mathrm{~ns}$

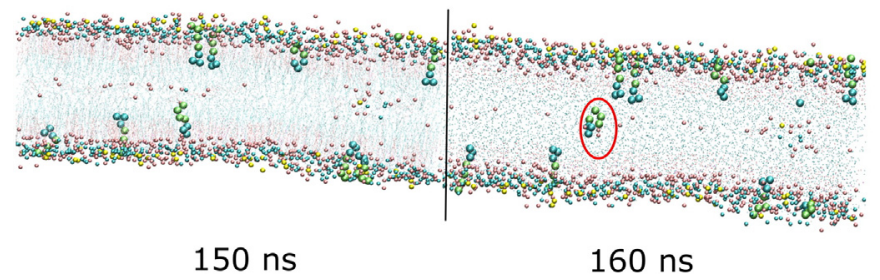

Fig. 8. Snapshots of the simulation at $0 \mathrm{~ns}, 30 \mathrm{~ns}, 150 \mathrm{~ns}$, and $160 \mathrm{~ns}$, respectively. Large spheres represent the retinyl acetate (RA). Lines and small spheres represent tail and head lipid chains. The region circled in red is the mobility of the RA between two lipids layers. Water is not represented. (For interpretation of the references to color in this figure legend, the reader is referred to the web version of this article.)
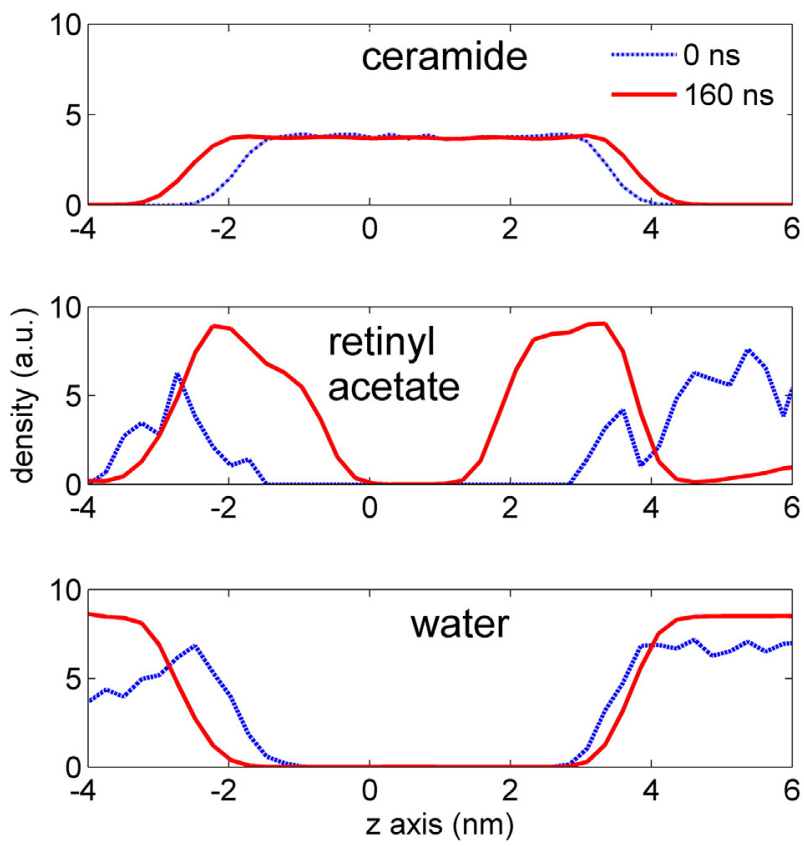

Fig. 9. Density profile for ceramide, cholesterol, retinyl acetate, and water at the beginning and end of the simulation.

C.A. Telléz S. would like to thank Capes/Brazil (88881.068140/2014-01 and 88887.125253/2015-00) for the grant. A.M. Cavaco-Paulo thanks Fapesp/Brazil (2014/05975-2) for the grant.

\section{References}

[1] M.E. Darvin, W. Sterry, J. Lademann, Resonance Raman spectroscopy as an effective tool for the determination of antioxidative stability of cosmetic formulations, J. Biophotonics 3 (2009) 82-88, http://dx.doi.org/10.1002/jbio.200910060.

[2] A.M. Kligman, G.L. Grove, R. Hirose, J.J. Leyden, Topical tretinoin for photoaged skin, J. Am. Acad. Dermatol. 15 (1986) 836-859, http://dx.doi.org/10.1016/S01909622(86)70242-9.

[3] W.J. Cunliffe, R. Caputo, B. Dreno, L. Förström, M. Heenen, C.E. Orfanos, Y. Privat, A.R. Aguilar, J. Meynadier, M. Alirezai, S. Jablonska, A. Shalita, J.S. Weiss, D.K. Chalker, C.N. Ellis, A. Greenspan, H.I. Katz, I. Kantor, L.E. Millikan, J.M. Swinehart, L. Swinyer, C. Whitmore, J. Czernielewski, M. Verschoore, Clinical efficacy and safety comparison of adapalene gel and tretinoin gel in the treatment of acne vulgaris: Europe and U.S. multicenter trials, J. Am. Acad. Dermatol. 36 (1997) S126-S134, http://dx.doi org/10.1016/S0190-9622(97)70056-2.

[4] P.E. Bermann, Aging skin: causes, treatments, and prevention, Nurs. Clin. North Am 42 (2007) 485-500, http://dx.doi.org/10.1016/j.cnur.2007.05.001.

[5] M. Manela-Azulay, E. Bagatin, Cosmeceuticals vitamins, Clin. Dermatol. 27 (2009) 469-474, http://dx.doi.org/10.1016/j.clindermatol.2009.05.010.

[6] K. Słoczyńska, A. Gunia-Krzyżak, D. Żelaszczyk, A.M. Waszkielewicz, H. Marona, Skin metabolism established with the use of MetaSite for selected retinoids employed in topical and systemic treatment of various skin disorders and found in cosmeceuticals, Acta Biochim. Pol. 62 (2015) 201-206, http://dx.doi.org/10.18388/ abp.2014_891.

[7] M. Ramos-e-Silva, L.R. Celem, S. Ramos-e-Silva, A.P. Fucci-da-Costa, Anti-aging cosmetics: facts and controversies, Clin. Dermatol. 31 (2013) 750-758, http://dx.doi. org/10.1016/j.clindermatol.2013.05.013.

[8] S. Arayachukeat, S.P. Wanichwecharungruang, T. Tree-Udom, Retinyl acetate-loaded nanoparticles: dermal penetration and release of the retinyl acetate, Int. J. Pharm. 404 (2011) 281-288, http://dx.doi.org/10.1016/j.ijpharm.2010.11.019.

[9] C. Das, M.G. Noro, P.D. Olmsted, Simulation studies of stratum corneum lipid mixtures, Biophys. J. 97 (2009) 1941-1951, http://dx.doi.org/10.1016/j.bpj. 2009.06.054.

[10] M. Egawa, T. Hirao, M. Takahashi, In vivo estimation of stratum corneum thickness from water concentration profiles obtained with Raman spectroscopy, Acta Derm. Venereol. 87 (2007) 4-8, http://dx.doi.org/10.2340/00015555-0183.

[11] J.M. Crowther, A. Sieg, P. Blenkiron, C. Marcott, P.J. Matts, J.R. Kaczvinsky, A.V Rawlings, Measuring the effects of topical moisturizers on changes in stratum corneum thickness, water gradients and hydration in vivo, Br. J. Dermatol. 159 (2008) 567-577, http://dx.doi.org/10.1111/j.1365-2133.2008.08703.x.

[12] J.T. Huzil, S. Sivaloganathan, M. Kohandel, M. Foldvari, I. Kotsireas, R. Melnik, B West, Modeling the Effects of Lipid Composition on Stratum Corneum Bilayers Using Molecular Dynamics Simulations, Adv. Math. Comput. Methods Addressing Mod. Challenges Sci. Technol. Soc., 2011 175-178, http://dx.doi.org/10.1063/1. 3663488.

[13] M.I. Hoopes, M.G. Noro, M.L. Longo, R. Faller, Bilayer structure and lipid dynamics in a model stratum corneum with oleic acid, J. Phys. Chem. B 115 (2011) 3164-3171, http://dx.doi.org/10.1021/jp109563s.

[14] C.A. Strott, Cholesterol sulfate in human physiology: what's it all about? J. Lipid Res. 44 (2003) 1268-1278, http://dx.doi.org/10.1194/jlr.R300005-JLR200.

[15] N. Noy, D.J. Kelleher, A.W. Scotto, Interactions of retinol with lipid bilayers: studies with vesicles of different radii, J. Lipid Res. 36 (1995) 375-382 (http://www.ncbi. nlm.nih.gov/pubmed/7751826).

[16] Y. Imai, X. Liu, J. Yamagishi, K. Mori, S. Neya, T. Hoshino, Computational analysis of water residence on ceramide and sphingomyelin bilayer membranes, J. Mol. Graph. Model. 29 (2010) 461-469, http://dx.doi.org/10.1016/j.jmgm.2010.09.002.

[17] J.A. Laszlo, K.O. Evans, D.L. Compton, M. Appell, Dihydrolipoyl dioleoylglycerol antioxidant capacity in phospholipid vesicles, Chem. Phys. Lipids 165 (2012) 160-168, http://dx.doi.org/10.1016/j.chemphyslip.2011.12.003.

[18] M. Venturoli, M.M. Sperotto, M. Kranenburg, B. Smit, Mesoscopic models of biological membranes, Phys. Rep. 437 (2006) 1-54, http://dx.doi.org/10.1016/j.physrep. 2006.07.006.

[19] S.M. Loverde, Molecular simulation of the transport of drugs across model membranes, J. Phys. Chem. Lett. 5 (2014) 1659-1665, http://dx.doi.org/10.1021/ jz500321d.

[20] S.J. Marrink, H.J. Risselada, S. Yefimov, D.P. Tieleman, A.H. de Vries, The MARTINI force field: coarse grained model for biomolecular simulations, J. Phys. Chem. B 111 (2007) 7812-7824, http://dx.doi.org/10.1021/jp071097f.

[21] H. Hwang, G.C. Schatz, M.A. Ratner, Coarse-grained molecular dynamics study of cyclic peptide nanotube insertion into a lipid bilayer †, J. Phys. Chem. A 113 (2009) 4780-4787, http://dx.doi.org/10.1021/jp8080657.

[22] H.I. Ingólfsson, C.A. Lopez, J.J. Uusitalo, D.H. de Jong, S.M. Gopal, X. Periole, S.] Marrink, The power of coarse graining in biomolecular simulations, Wiley Interdiscip, Rev. Comput. Mol. Sci. 4 (2014) 225-248, http://dx.doi.org/10.1002/ wcms.1169.

[23] M. Martins, N.G. Azoia, A. Ribeiro, U. Shimanovich, C. Silva, A. Cavaco-Paulo, In vitro and computational studies of transdermal perfusion of nanoformulations containing a large molecular weight protein, Colloids Surf. B: Biointerfaces 108 (2013) 271-278, http://dx.doi.org/10.1016/j.colsurfb.2013.02.032.

[24] A.P. Teixeira, J.L. Rangel, L.J. Raniero, M.G. Tosato, P.P. Fávero, A.A. Martin, Confocal Raman spectroscopy: determination of natural moisturizing factor profile related to skin hydration, Rev. Bras. Eng. Biomédica. 30 (2014) 11-16, http://dx.doi.org/ 10.4322/rbeb.2014.002.

[25] M.G. Tosato, R.S. Alves, E.A.P. dos Santos, L. Raniero, P.F.C. Menezes, K.M.S. Belletti, C.E.O. Praes, A.A. Martin, Raman spectroscopic investigation of the effects of cosmetic formulations on the constituents and properties of human skin, Photomed. Laser Surg. 30 (2) (2012) 85-91.

[26] P.D.A. Pudney, M. Mélot, P.J. Caspers, A. Van Der Pol, G.J. Puppels, An in vivo confocal Raman study of the delivery of trans retinol to the skin, Appl. Spectrosc. 61 (2007) 804-811, http://dx.doi.org/10.1366/000370207781540042.

[27] H.L. Royden, P. Fitzpatrick, Real Analysis, Prentice Hall, New York, 1988.

[28] P.J. Caspers, G.W. Lucassen, R. Wolthuis, H.A. Bruining, G.J. Puppels, In vitro and in vivo Raman spectroscopy of human skin, Biospectroscopy 4 (1998) S31-S39, http://dx.doi.org/10.1002/(SICI)1520-6343.

[29] P.J. Caspers, A.C. Williams, E.A. Carter, H.G.M. Edwards, B.W. Barry, H.A. Bruining, G.J Puppels, Monitoring the penetration enhancer dimethyl sulfoxide in human stratum corneum in vivo by confocal Raman spectroscopy, Pharm. Res. 19 (10) (2002) 1577-1580. 
[30] B. Mogilevych, D. Isensee, J.L. Rangel, C. Dal Pizzol, V.C.A. Martinello, G.C. Dieamant, A.A. Martin, Study of the vitamins A, E and C esters penetration into the skin by confocal Raman spectroscopy in vivo, in: C. Kurachi, K. Svanberg, B.J. Tromberg, V.S. Bagnato (Eds.),Prog. Biomed. Opt. Imaging - Proc. SPIE, 2015, p. 95312A, http:// dx.doi.org/10.1117/12.2181016.

[31] J.O. Morales, K. Valdés, J. Morales, F. Oyarzun-Ampuero, Lipid nanoparticles for the topical delivery of retinoids and derivatives, Nanomedicine 10 (2015) 253-269, http://dx.doi.org/10.2217/nnm.14.159.

[32] P.V. Pople, K.K. Singh, Development and evaluation of topical formulation containing solid lipid nanoparticles of vitamin A, AAPS PharmSciTech. 7 (2006) E63-E69, http://dx.doi.org/10.1208/pt070491.

[33] N. Nakagawa, M. Matsumoto, S. Sakai, In vivo measurement of the water content in the dermis by confocal Raman spectroscopy, Skin Res. Technol. 16 (2010) 137-141, http://dx.doi.org/10.1111/j.1600-0846.2009.00410.x.

[34] M. Mélot, P.D.A. Pudney, A.-M. Williamson, P.J. Caspers, A. Van Der Pol, G.J. Puppels, Studying the effectiveness of penetration enhancers to deliver retinol through the stratum cornum by in vivo confocal Raman spectroscopy, J. Control. Release 138 (2009) 32-39, http://dx.doi.org/10.1016/j.jconrel.2009.04.023.

[35] D. Karthigeyan, S. Siddhanta, A.H. Kishore, S.S.R.R. Perumal, H. Agren, S. Sudevan, A.V. Bhat, K. Balasubramanyam, R.K. Subbegowda, T.K. Kundu, C. Narayana, SERS and MD simulation studies of a kinase inhibitor demonstrate the emergence of a potential drug discovery tool, Proc. Natl. Acad. Sci. 111 (2014) 10416-10421, http://dx. doi.org/10.1073/pnas.1402695111.

[36] R. Notman, J. Anwar, Breaching the skin barrier - insights from molecular simulation of model membranes, Adv. Drug Deliv. Rev. 65 (2013) 237-250, http://dx.doi. org/10.1016/j.addr.2012.02.011.

[37] J. Zbytovská, M.A. Kiselev, S.S. Funari, V.M. Garamus, S. Wartewig, K. Palát, R Neubert, Influence of cholesterol on the structure of stratum corneum lipid mode membrane, Colloids Surf. A Physicochem. Eng. Asp. 328 (2008) 90-99, http://dx. doi.org/10.1016/j.colsurfa.2008.06.032.

[38] J. Sato, M. Denda, J. Nakanishi, J. Nomura, J. Koyama, Cholesterol sulfate inhibits proteases that are involved in desquamation of stratum corneum, J. Invest. Dermatol. 111 (1998) 189-193, http://dx.doi.org/10.1046/j.1523-1747.1998.00244.x.

[39] B. Sommer, T. Dingersen, C. Gamroth, S.E. Schneider, S. Rubert, J. Krüger, K.-J. Dietz, CELLmicrocosmos 2.2 MembraneEditor: a modular interactive shape-based software approach to solve heterogeneous membrane packing problems, J. Chem. Inf. Model. 51 (2011), 110419120935062. http://dx.doi.org/10.1021/ci1003619.

[40] S. Pronk, S. Pall, R. Schulz, P. Larsson, P. Bjelkmar, R. Apostolov, M.R. Shirts, J.C. Smith, P.M. Kasson, D. van der Spoel, B. Hess, E. Lindahl, GROMACS 4.5: a high-throughput and highly parallel open source molecular simulation toolkit, Bioinformatics. 29 (2013) 845-854, http://dx.doi.org/10.1093/bioinformatics/btt055.

[41] B. Hess, P-LINCS: a parallel linear constraint solver for molecular simulation, J. Chem. Theory Comput. 4 (2008) 116-122, http://dx.doi.org/10.1021/ct700200b.

[42] H.J.C. Berendsen, J.P.M. Postma, W.F. van Gunsteren, A. DiNola, J.R. Haak, Molecular dynamics with coupling to an external bath, J. Chem. Phys. 81 (1984) 3684, http://dx.doi.org/10.1063/1.448118.

[43] G. Bussi, D. Donadio, M. Parrinello, Canonical sampling through velocity rescaling, J. Chem. Phys. 126 (2007)http://dx.doi.org/10.1063/1.2408420.

[44] A.J. Rzepiela, L.V. Schäfer, N. Goga, H.J. Risselada, A.H. De Vries, S.J. Marrink, Reconstruction of atomistic details from coarse-grained structures, J. Comput. Chem. 31 (2010) NA, http://dx.doi.org/10.1002/jcc.21415.

[45] A. Choubey, R.K. Kalia, N. Malmstadt, A. Nakano, P. Vashishta, Cholesterol translocation in a phospholipid membrane, Biophys. J. 104 (2013) 2429-2436, http://dx.doi. org/10.1016/j.bpj.2013.04.036.

[46] D.W. Mayo, F.A. Miller, R.W. Hannah, Course Notes on the Interpretation of Infrared and Raman Spectra, 2004http://dx.doi.org/10.1002/0471690082. 